\title{
Convenient synthesis of the 2,5-di-substituted 1,3,4- oxadiazole derivatives under microwave
}

\author{
Dipti L. Namera, Umed C. Bhoya* \\ Chemical Research Laboratory, Department of Chemistry, Saurashtra University, \\ Rajkot - 360005, Gujrat, India \\ *E-mail address: drucbhoya@gmail.com
}

\begin{abstract}
We have reported some novel 1,3,4-oxadiazole synthesized by conventional method as well as microwave assisted method. The reaction of different substituted cinnamic acid 2a-o with 2-(4chlorophenyl) acetohydrazide by using phosphoric anhydride as catalyst, yielded a series of 2,5-disubstituted 1,3,4-oxadiazole 6a-o. The structures of all synthesized compounds are well characterized by Mass, FT-IR, ${ }^{1} \mathrm{H}$ NMR, ${ }^{13} \mathrm{C}$ NMR and elemental analysis. After obtaining experimental data regarding the yield and the time taken for the synthesis by both the methods, conventional and microwave assisted method, it was proved that the microwave assisted method is convenient for synthesis of this type of 2,5-di-substituted 1,3,4-oxadiazole 6a-o.
\end{abstract}

Keywords: Substituted cinnamic acid; Substituted 1,3,4-oxadiazoles; phosphoric anhydride

\section{INTRODUCTION}

In the field of synthetic organic chemistry, major challenges are to develop the new method for the synthesis of five member heterocyclic compounds. Literature survey reveals five member 1,3,4-oxadiazole derivatives, which belong to an important group of heterocyclic compounds, have been the issue of wide-ranging study in the recent time. Various reports have displayed their chemistry and use [1-3].

A Wide variety of substituted 1,3,4-oxadiazoles have attracted considerable attention in the field of drug discovery because of their wide range of pharmacological activities, including anti-proliferative [4], antifungal [5], antibacterial [6,7], anticancer [8], antitubercular [9], GABA ${ }_{A}$ receptor agonists [10], anti-inflammatory [11], anti HIV [12]. Different methods have been reported for the synthesis of 1,3,4-oxadiazoles involving cyclization of 1,4-di-substituted thiosemicarbazide in the presence dicyclohexylcarbodiimide (DCC) [13].

Several cyclodehydrating agents such as $\mathrm{Et}_{2} \mathrm{O}-\mathrm{BF}_{3}$, triflic anhydride, thionyl chloride, polyphosphoric acid, 1,1,1,3,3,3-hexamethyldisilazane, sulfuric acid and phosphorus oxychloride, have been used.

However, some of these newer reported also suffer from drawbacks such as they require to heat reactants for an extended periodic time at elevated temperatures, awkward product isolation procedure, and environmental pollution. Moreover, the yield is frequently only moderate or low. 
In previous we have reported this type of compounds by using phosphorous oxychloride [14], but we have observed that use of phosporic anhydried is better than phosphorous oxychloride. In view of the above-mentioned findings, the purpose of the present work was to investigate the new methodologies for the one pot synthesis of $1,3,4-$ oxadiazole with high yield and lesser reaction time.

\section{MATERIAL AND METHOD}

Melting points were determined in open glass capillaries. Infrared spectra were recorded on a Shimadzu FT-IR-8400 spectrometer using KBr pellet method. ${ }^{1} \mathrm{H}$ NMR and ${ }^{13} \mathrm{C}$ NMR spectra were recorded on a Bruker Advance $400 \mathrm{MHz}$ NMR Spectrometer using DMSO- $\mathrm{d}_{6}$ as a solvent and TMS as a Internal standard. The type of signal is indicated by following letter: $\mathrm{s}=$ singlet, $\mathrm{d}=$ doublet, $\mathrm{t}=$ triplet, $\mathrm{dd}=$ doublet of doublet, $\mathrm{q}=$ quartet, $\mathrm{m}=$ multiplet. Mass spectra were recorded on Shimadzu GC-MS-QP-2010 model using Direct Injection Probe technique. Microanalysis was performed on Euro EA Elemental Analyser. Reaction was monitored by thin-layer chromatography (TLC).

A mixture of different substituted aromatic aldehyde $(0.01 \mathrm{~mol})$ and malonic acid $(0.015 \mathrm{~mol})$ was taken in pyridine $(15 \mathrm{ml})$ and catalytic amount of piperidine was added. The reaction mixture was refluxed for 6 hours. After the completion of reaction, the reaction mass was poured on to the crushed ice: $\mathrm{HCl}(1: 1)$ solution. Filtered the separated product and wash with diluted $\mathrm{HCl}$ and crystallized from methanol. The product was enough pure and taken for next step without further purification. Yield $75 \%$ to $88 \%$.

A mixture of 2-(4-chlorophenyl) acetic acid was taken in methanol and adds few drops of $\mathrm{H}_{2} \mathrm{SO}_{4}$ and refluxes it about 8 hours. Then cool the reaction mixture and poured into crushed ice, filter the separated product and use it for further step without purification.

A mixture of 2-(4-chlorophenyl) acetic ester $(0.1 \mathrm{~mol})$ and hydrazine hydrate $(20 \mathrm{ml})$ was stirred at room temperature for 4 hours. TLC using solvent system Chloroform monitored the reaction progress: methanol (9:1). Separated product was filtered and washed with water. Recrystallized from acetic acid. Yield was $83 \%$

\section{1. General procedure for the synthesis of (E)-2-(4-chlorobenzyl)-5-substituted styryl-1,3,4-oxadiazole; 6(a-o)}

The compound $5(0.01 \mathrm{~mol})$, compound 2(a-o) $(0.01 \mathrm{~mol})$ and phosphoric anhydride $(0.015 \mathrm{~mol})$ were taken in $150 \mathrm{ml} \mathrm{RBF}$ and heated at $600 \mathrm{~W}$ in microwave for 3-6 minutes. Completion of reaction was checked and monitered by thin leyer chromatography. After the completion of reaction, reaction mixture was cooled and poured in to crushed ice, stirred for 30 minutes and filtered.

The obtained solid mass was further washed with $50 \mathrm{ml} 10 \%$ solution of sodium bicarbonate and followed by wash with $50 \mathrm{ml}$ deminaralized water. The resulting compound was purified by column chromatography by silica gel 230-400 mesh using ethyl acetate: hexane (4: $6 \mathrm{v} / \mathrm{v})$ as eluent. Yield : $75 \%$. 


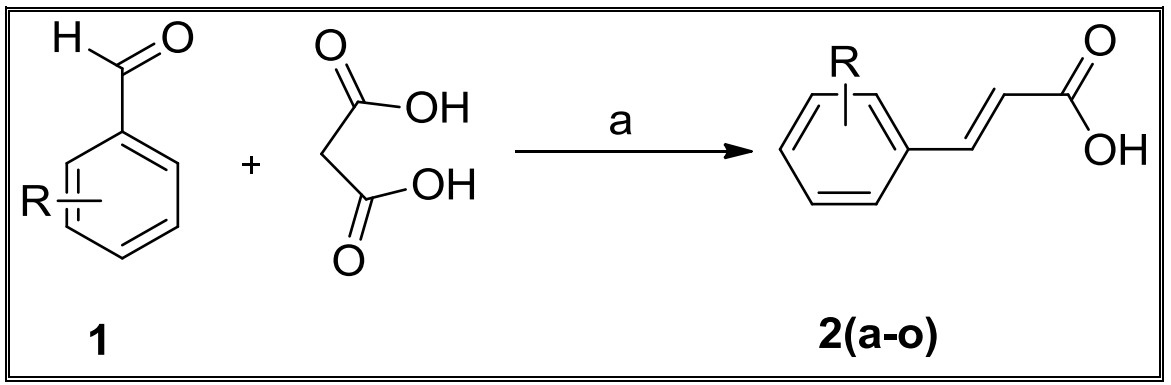

Scheme 1

a) Pyridine, Pipyridine (Catalyst), reflux for 6 hrs.

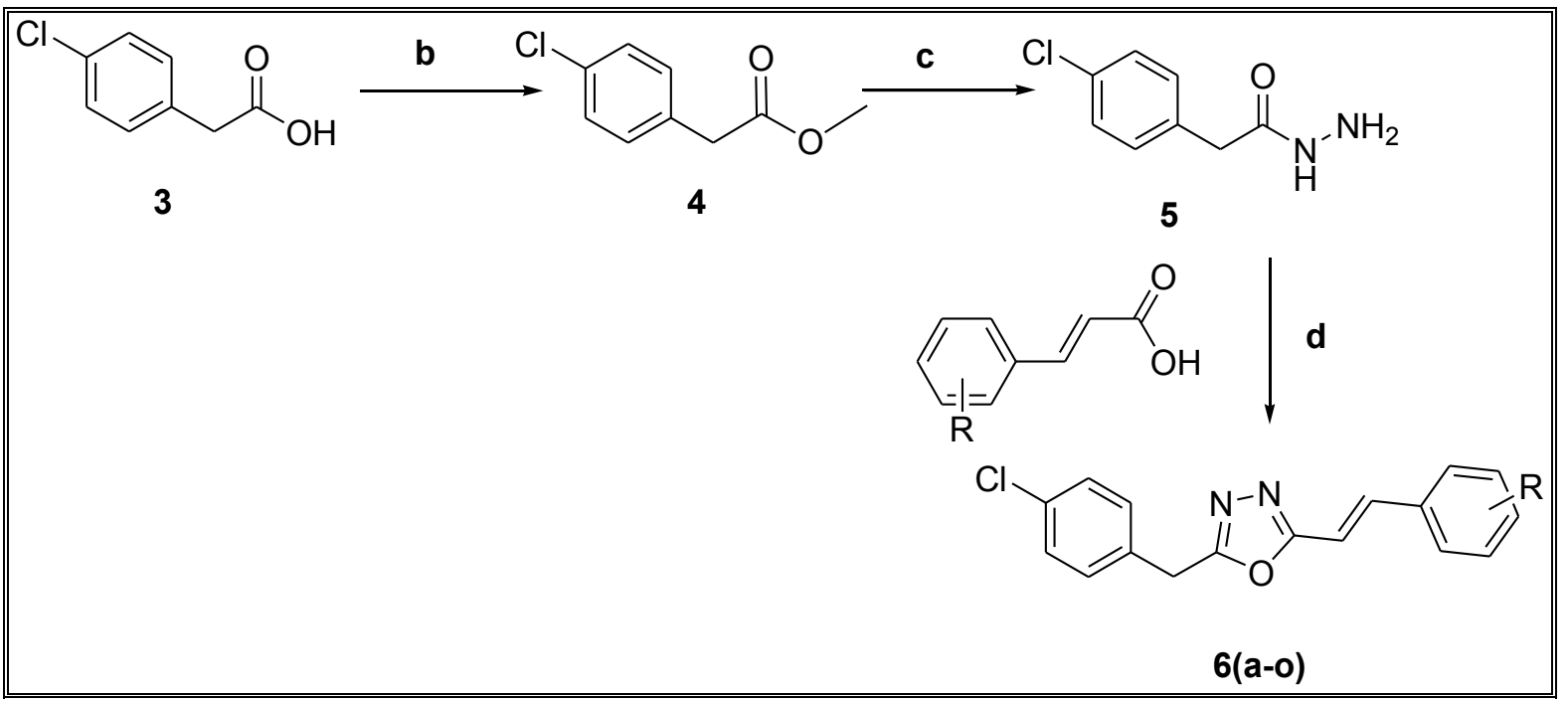

Scheme 2

b) $\mathrm{MeOH}, \mathrm{H}_{2} \mathrm{SO}_{4}, 8$ hrs., c) $\mathrm{NH}_{2} \mathrm{NH}_{2}$, rt, 4 hrs., d) Phosphoric anhydride, Reflux for 7-9 hour or microwave irradiation (3-6 min).

\section{SPECTRAL DATA}

2-(4-chlorobenzyl)-5-styryl-1,3,4-oxadiazole; (6a)

MP: $144-146{ }^{\circ} \mathrm{C}$; IR $\left(\mathrm{cm}^{-1}\right): 3048\left(\mathrm{Ar}-\mathrm{H}\right.$ stretch), $2850\left(\mathrm{CH}_{2}\right.$-stretch), $1684(\mathrm{C}=\mathrm{N}$ stretch of 1,3,4-oxadiazole ring), $2140(\mathrm{C}=\mathrm{C}$ stretching $), 1252(\mathrm{C}-\mathrm{O}-\mathrm{C}$ stretch of 1,3,4-oxadiazole ring); MS: $\mathrm{m} / \mathrm{z}=296 ;{ }^{1} \mathrm{H}$ NMR $\left(\mathrm{CDCl}_{3}\right) \delta \mathrm{ppm}: 3.89(2 \mathrm{H}, \mathrm{s}), 6.80(1 \mathrm{H}, \mathrm{s}), 7.12(1 \mathrm{H}, \mathrm{s}), 7.32$ $(2 \mathrm{H}, \mathrm{s}), 7.31-7.52(5 \mathrm{H}, \mathrm{m}), 7.17-7.35(4 \mathrm{H}, \mathrm{m})$ Elemental Analysis: Calcd. For $\mathrm{C}_{17} \mathrm{H}_{13} \mathrm{ClN}_{2} \mathrm{O}$; C, 68.81 ; H, 4.42; N, 9.44; O, 5.39 Found: C, 67.81; H, 4.50; N, 10.12; O, 6.21

\section{2-(4-chlorobenzyl)-5-(2, 4-dimethoxystyryl)-1,3,4-oxadiazole; (6b)}

MP: $132-134{ }^{\circ} \mathrm{C}$; IR $\left(\mathrm{cm}^{-1}\right)$ : $3052(\mathrm{Ar}-\mathrm{H}$ stretch $), 2858\left(\mathrm{CH}_{2}\right.$-stretch $), 1678(\mathrm{C}=\mathrm{N}$ stretch of 1,3,4-oxadiazole ring), $2146(\mathrm{C}=\mathrm{C}$ stretching), $1254(\mathrm{C}-\mathrm{O}-\mathrm{C}$ stretch of 1,3,4-oxadiazole ring), $1078\left(\mathrm{C}-\mathrm{O}\right.$ stretch); $\mathrm{MS}: \mathrm{m} / \mathrm{z}=356 ;{ }^{1} \mathrm{H}$ NMR $\left(\mathrm{CDCl}_{3}\right) \delta \mathrm{ppm}: 3.74(1 \mathrm{H}, \mathrm{s}), 3.80(6 \mathrm{H}, \mathrm{s})$, $6.61(1 \mathrm{H}, \mathrm{s}), 6.95(1 \mathrm{H}, \mathrm{s}), 7.12(1 \mathrm{H}, \mathrm{s}), 7.17(1 \mathrm{H}, \mathrm{s}), 6.58-6.78(3 \mathrm{H}, \mathrm{m})$; Elemental Analysis: Calcd. For $\mathrm{C}_{19} \mathrm{H}_{17} \mathrm{ClN}_{2} \mathrm{O}_{3} ; \mathrm{C}, 63.96 ; \mathrm{H}, 4.80 ; \mathrm{Cl}, 9.94 ; \mathrm{N}, 7.85 ; \mathrm{O}, 13.45$ Found: $\mathrm{C}, 64.46 ; \mathrm{H}$, $5.72 ; \mathrm{Cl}, 8.64 ; \mathrm{N}, 7.28 ; \mathrm{O}, 14.05$ 


\section{2-(4-chlorobenzyl)-5-(4-nitrostyryl)-1,3,4-oxadiazole; (6c)}

MP: $162-164{ }^{\circ} \mathrm{C}$; IR $\left(\mathrm{cm}^{-1}\right)$ : $3054(\mathrm{Ar}-\mathrm{H}$ stretch $), 2860\left(\mathrm{CH}_{2}\right.$-stretch $), 1686(\mathrm{C}=\mathrm{N}$ stretch of 1,3,4-oxadiazole ring), $2166(\mathrm{C}=\mathrm{C}$ stretching), $1255(\mathrm{C}-\mathrm{O}-\mathrm{C}$ stretch of 1,3,4-oxadiazole ring), 1089 (C-O stretch); 1366 (Assymetric $\mathrm{N}-\mathrm{O}$ stretch of $\mathrm{C}-\mathrm{NO}_{2}$ ) MS: $\mathrm{m} / \mathrm{z}=341 ;{ }^{1} \mathrm{H}$ NMR $\left(\mathrm{CDCl}_{3}\right) \delta$ ppm: $3.75(2 \mathrm{H}, \mathrm{s}), 7.04(1 \mathrm{H}, \mathrm{s}), 7.14(1 \mathrm{H}, \mathrm{s}), 7.14-7.25(4 \mathrm{H}, \mathrm{m}), 8.20-8.37$ $(4 \mathrm{H}, \mathrm{m})$; Elemental Analysis: Calcd. For $\mathrm{C}_{17} \mathrm{H}_{12} \mathrm{ClN}_{3} \mathrm{O}_{3} ; \mathrm{C}, 59.75 ; \mathrm{H}, 3.54 ; \mathrm{Cl}, 10.37 ; \mathrm{N}$, 12.30; O, 14.04: Found:- C, 58.65; H, 4.44; Cl, 9.78; N, 12.15; O, 15.15

\section{2-(4-chlorobenzyl)-5-(4-fluorostyryl)-1,3,4-oxadiazole; (6d)}

MP: $140-142{ }^{\circ} \mathrm{C}$; IR $\left(\mathrm{cm}^{-1}\right): 3045$ (Ar-H stretch), $2856\left(\mathrm{CH}_{2}\right.$-stretch), $1670(\mathrm{C}=\mathrm{N}$ stretch of 1,3,4-oxadiazole ring), $2148(\mathrm{C}=\mathrm{C}$ stretching $), 1262(\mathrm{C}-\mathrm{O}-\mathrm{C}$ stretch of 1,3,4-oxadiazole ring), 1080 (C-O stretch); MS: $\mathrm{m} / \mathrm{z}=314 ;{ }^{1} \mathrm{H}$ NMR $\left(\mathrm{CDCl}_{3}\right) \delta \mathrm{ppm:} 3.65(2 \mathrm{H}, \mathrm{s}), 6.95(1 \mathrm{H}, \mathrm{s})$, 7.01 $(1 \mathrm{H}, \mathrm{s}), 7.15(2 \mathrm{H}, \mathrm{s}), 7.36(2 \mathrm{H}, \mathrm{s}), 7.18-7.62(4 \mathrm{H}, \mathrm{m})$; Elemental Analysis: Calcd. For $\mathrm{C}_{17} \mathrm{H}_{2} \mathrm{ClFN}_{2} \mathrm{O} ; \mathrm{C}, 64.87 ; \mathrm{H}, 3.84 ; \mathrm{Cl}, 11.26 ; \mathrm{F}, 6.04 ; \mathrm{N}, 8.90 ; \mathrm{O}, 5.08$ : Found: C, 64.61; H, $4.98 ; \mathrm{Cl}, 10.85 ; \mathrm{F}, 6.86 ; \mathrm{N}, 8.12 ; \mathrm{O}, 6.14$

\section{2-(4-chlorobenzyl)-5-(4-methoxystyryl)-1,3,4-oxadiazole; (6e)}

MP: $136-138^{\circ} \mathrm{C}$; IR $\left(\mathrm{cm}^{-1}\right)$ : $3060(\mathrm{Ar}-\mathrm{H}$ stretch $), 2862\left(\mathrm{CH}_{2}\right.$-stretch $), 1676(\mathrm{C}=\mathrm{N}$ stretch of 1,3,4-oxadiazole ring), $2152(\mathrm{C}=\mathrm{C}$ stretching), $1250(\mathrm{C}-\mathrm{O}-\mathrm{C}$ stretch of 1,3,4-oxadiazole ring), $1080\left(\mathrm{C}-\mathrm{O}\right.$ stretch); $\mathrm{MS}: \mathrm{m} / \mathrm{z}=326 ;{ }^{1} \mathrm{H}$ NMR $\left(\mathrm{CDCl}_{3}\right) \delta$ ppm: $3.56(2 \mathrm{H}, \mathrm{s})$, 6.91 $(1 \mathrm{H}, \mathrm{s}), 7.06(1 \mathrm{H}, \mathrm{s}), 7.18(2 \mathrm{H}, \mathrm{s}), 7.40(2 \mathrm{H}, \mathrm{s}), 7.24-7.50(4 \mathrm{H}, \mathrm{m})$; Elemental Analysis: Calcd. For $\mathrm{C}_{18} \mathrm{H}_{15} \mathrm{ClN}_{2} \mathrm{O}_{2} ; \mathrm{C}, 66.16 ; \mathrm{H}, 4.63 ; \mathrm{Cl}, 10.85 ; \mathrm{N}, 8.57 ; \mathrm{O}, 9.79$ : Found: C, 67.08; H, 5.83; $\mathrm{Cl}, 9.98 ; \mathrm{N}, 9.32 ; \mathrm{O}, 10.61$

\section{2-(4-chlorobenzyl)-5-(4-methylstyryl)-1,3,4-oxadiazole; (6f)}

MP: $130-132{ }^{\circ} \mathrm{C}$; IR $\left(\mathrm{cm}^{-1}\right)$ : 3062 (Ar-H stretch), $2859\left(\mathrm{CH}_{2}\right.$-stretch), $1680(\mathrm{C}=\mathrm{N}$ stretch of 1,3,4-oxadiazole ring), $2159(\mathrm{C}=\mathrm{C}$ stretching), $1260(\mathrm{C}-\mathrm{O}-\mathrm{C}$ stretch of 1,3,4-oxadiazole ring), $1087\left(\mathrm{C}-\mathrm{O}\right.$ stretch); $\mathrm{MS}: \mathrm{m} / \mathrm{z}=310 ;{ }^{1} \mathrm{H}$ NMR $\left(\mathrm{CDCl}_{3}\right) \delta \mathrm{ppm}: 2.35(3 \mathrm{H}, \mathrm{s}), 3.55(2 \mathrm{H}, \mathrm{s})$, $6.98(1 \mathrm{H}, \mathrm{s}), 7.04(1 \mathrm{H}, \mathrm{s}), 7.17-7.45(4 \mathrm{H}, \mathrm{m}), 7.50-7.63(4 \mathrm{H}, \mathrm{m})$; Elemental Analysis: Calcd. For $\mathrm{C}_{18} \mathrm{H}_{15} \mathrm{ClN}_{2} \mathrm{O}$ : C, 69.57; H, 4.86; Cl, 11.41; N, 9.01; O, 5.15: Found: C, 69.18; H, 6.06; $\mathrm{Cl}, 10.96 ; \mathrm{N}, 9.78 ; \mathrm{O}, 6.28$

\section{2-(4-chlorobenzyl)-5-(4-hexyloxystyryl)-1,3,4-oxadiazole; (6g)}

MP: $120-122{ }^{\circ} \mathrm{C}$; IR $\left(\mathrm{cm}^{-1}\right): 3058$ (Ar-H stretch), $2864\left(\mathrm{CH}_{2}\right.$-stretch), $1683(\mathrm{C}=\mathrm{N}$ stretch of 1,3,4-oxadiazole ring), $2150(\mathrm{C}=\mathrm{C}$ stretching), $1250(\mathrm{C}-\mathrm{O}-\mathrm{C}$ stretch of 1,3,4-oxadiazole ring), $1072(\mathrm{C}-\mathrm{O}$ stretch $) ; \mathrm{MS}: \mathrm{m} / \mathrm{z}=422 ;{ }^{1} \mathrm{H}$ NMR $\left(\mathrm{CDCl}_{3}\right) \delta$ ppm: $1.09(3 \mathrm{H}, \mathrm{t}), 1.42$ $(2 \mathrm{H}, \mathrm{m}), 1.58-1.78(6 \mathrm{H}, \mathrm{m}), 4.37(2 \mathrm{H}, \mathrm{t}), 6.95(1 \mathrm{H}, \mathrm{s}), 7.02(1 \mathrm{H}, \mathrm{s}), 7.12-7.34(4 \mathrm{H}, \mathrm{m}), 7.67-7.80$ $(4 \mathrm{H}, \mathrm{m})$; Elemental Analysis: Calcd. For $\mathrm{C}_{25} \mathrm{H}_{27} \mathrm{ClN}_{2} \mathrm{O}_{2} ; \mathrm{C}, 70.99 ; \mathrm{H}, 6.43 ; \mathrm{Cl}, 8.38 ; \mathrm{N}, 6.62$; O, 7.57: Found: C, 72.02; H, 6.89; Cl, 8.14; N, 6.32; O, 8.45

\section{2-(4-chlorobenzyl)-5-(4-chlorostyryl)-1,3,4-oxadiazole; (6h)}

MP: $138-140{ }^{\circ} \mathrm{C}$; IR $\left(\mathrm{cm}^{-1}\right)$ : $3048(\mathrm{Ar}-\mathrm{H}$ stretch $), 2860\left(\mathrm{CH}_{2}\right.$-stretch $), 1685(\mathrm{C}=\mathrm{N}$ stretch of 1,3,4-oxadiazole ring), $2146(\mathrm{C}=\mathrm{C}$ stretching $), 1262(\mathrm{C}-\mathrm{O}-\mathrm{C}$ stretch of 1,3,4-oxadiazole ring), $1068\left(\mathrm{C}-\mathrm{O}\right.$ stretch); $\mathrm{MS}: \mathrm{m} / \mathrm{z}=330 ;{ }^{1} \mathrm{H}$ NMR $\left(\mathrm{CDCl}_{3}\right) \delta$ ppm: $3.55(2 \mathrm{H}, \mathrm{s}), 6.86$ $(1 \mathrm{H}, \mathrm{s}), 7.10(1 \mathrm{H}, \mathrm{s}), 7.20(2 \mathrm{H}, \mathrm{s}), 7.34(2 \mathrm{H}, \mathrm{s}), 7.45-7.62(4 \mathrm{H}, \mathrm{m})$; Elemental Analysis: Calcd. For $\mathrm{C}_{17} \mathrm{H}_{12} \mathrm{C}_{12} \mathrm{~N}_{2} \mathrm{O} ; \mathrm{C}, 61.65 ; \mathrm{H}, 3.65 ; \mathrm{Cl}, 21.41 ; \mathrm{N}, 8.46 ; \mathrm{O}, 4.83$ : Found: $\mathrm{C}, 61.58 ; \mathrm{H}, 5.86$; $\mathrm{Cl}, 19.82 ; \mathrm{N}, 9.98 ; \mathrm{O}, 5.68$

\section{2-(4-chlorobenzyl)-5-(2,5-dimethoxystyryl)-1,3,4-oxadiazole; 6i)}

MP: $124-126{ }^{\circ} \mathrm{C}$; IR $\left(\mathrm{cm}^{-1}\right): 3052(\mathrm{Ar}-\mathrm{H}$ stretch$), 2858\left(\mathrm{CH}_{2}\right.$-stretch) $1682(\mathrm{C}=\mathrm{N}$ stretch of 1,3,4-oxadiazole ring), $2158(\mathrm{C}=\mathrm{C}$ stretching), $1262(\mathrm{C}-\mathrm{O}-\mathrm{C}$ stretch of 1,3,4-oxadiazole ring), $1056\left(\mathrm{C}-\mathrm{O}\right.$ stretch); $\mathrm{MS}: \mathrm{m} / \mathrm{z}=356 ;{ }^{1} \mathrm{H} \mathrm{NMR}\left(\mathrm{CDCl}_{3}\right) \delta \mathrm{ppm}: 3.64(1 \mathrm{H}, \mathrm{s}), 3.78(6 \mathrm{H}, \mathrm{s})$, 
$6.71(1 \mathrm{H}, \mathrm{s}), 6.88(1 \mathrm{H}, \mathrm{s}), 7.13(1 \mathrm{H}, \mathrm{s}), 7.18(1 \mathrm{H}, \mathrm{s}), 6.68-6.86(3 \mathrm{H}, \mathrm{m})$; Elemental Analysis: Calcd. For $\mathrm{C}_{19} \mathrm{H}_{17} \mathrm{ClN}_{2} \mathrm{O}_{3} ; \mathrm{C}, 63.96 ; \mathrm{H}, 4.80 ; \mathrm{Cl}, 9.94 ; \mathrm{N}, 7.85 ; \mathrm{O}, 13.45$ : Found: $\mathrm{C}, 64.08 ; \mathrm{H}$, 5.78; $\mathrm{Cl}, 9.86 ; \mathrm{N}, 8.78 ; \mathrm{O}, 15.42$

\section{2-(4-chlorobenzyl)-5-(3,4,5-trimethoxystyryl)-1,3,4-oxadiazole; (6j)}

MP: $120-122^{\circ} \mathrm{C}$; IR $\left(\mathrm{cm}^{-1}\right)$ : $3105(\mathrm{Ar}-\mathrm{H}$ stretch $), 2856\left(\mathrm{CH}_{2}\right.$-stretch $), 1680(\mathrm{C}=\mathrm{N}$ stretch of 1,3,4-oxadiazole ring), $2178(\mathrm{C}=\mathrm{C}$ stretching), $1283(\mathrm{C}-\mathrm{O}-\mathrm{C}$ stretch of 1,3,4-oxadiazole ring), $1120\left(\mathrm{C}-\mathrm{O}\right.$ stretch); $\mathrm{MS}: \mathrm{m} / \mathrm{z}=386 ;{ }^{1} \mathrm{H}$ NMR $\left(\mathrm{CDCl}_{3}\right) \delta$ ppm: $3.79(2 \mathrm{H}, \mathrm{s}), 3.84$ $(9 \mathrm{H}, \mathrm{s}), 6.76(2 \mathrm{H}, \mathrm{s}), 6.98(1 \mathrm{H}, \mathrm{s}), 6.99(1 \mathrm{H}, \mathrm{s}), 7.20-7.47(4 \mathrm{H}, \mathrm{m}) ;$ Elemental Analysis: Calcd. For $\mathrm{C}_{20} \mathrm{H}_{19} \mathrm{ClN}_{6.2} \mathrm{O}_{4} ; \mathrm{C}, 62.10 ; \mathrm{H}, 4.95 ; \mathrm{Cl}, 9.17 ; \mathrm{N}, 7.24 ; \mathrm{O}, 16.54$ Found: $\mathrm{C}, 62.84 ; \mathrm{H}, 5.98$; $\mathrm{Cl}, 9.08 ; \mathrm{N}, 8.24 ; \mathrm{O}, 17.24$

\section{5-(2-(5-(4-chlorobenzyl)-1,3,4-oxadiazol-2-yl)vinyl)-2-methoxyphenol; (6k)}

MP: $142-144{ }^{\circ} \mathrm{C}$; IR $\left(\mathrm{cm}^{-1}\right)$ : $3078\left(\mathrm{Ar}-\mathrm{H}\right.$ stretch), $2852\left(\mathrm{CH}_{2}\right.$-stretch), $1665(\mathrm{C}=\mathrm{N}$ stretch of 1,3,4-oxadiazole ring), $2148(\mathrm{C}=\mathrm{C}$ stretching), $1268(\mathrm{C}-\mathrm{O}-\mathrm{C}$ stretch of 1,3,4-oxadiazole ring), $1104\left(\mathrm{C}-\mathrm{O}\right.$ stretch) $\mathrm{MS}: \mathrm{m} / \mathrm{z}=342 ;{ }^{1} \mathrm{H}$ NMR $\left(\mathrm{CDCl}_{3}\right) \delta \mathrm{ppm:} 3.80(2 \mathrm{H}, \mathrm{s}), 5.17(1 \mathrm{H}, \mathrm{s})$, 6.79(1H,s), $6.88(1 \mathrm{H}, \mathrm{s}), 7.28-7.67(4 \mathrm{H}, \mathrm{m})$; Elemental Analysis: Calcd. For $\mathrm{C}_{18} \mathrm{H}_{15} \mathrm{ClN}_{2} \mathrm{O}_{3} ; \mathrm{C}$, 63.07; H, 4.41; Cl, 10.34; N, 8.17; O, 14.00 Found: C, 64.98; H, 5.84; Cl, 10.45; N, 9.17; O, 14.68

\section{2-(4-chlorobenzyl)-5-(2-fluorostyryl)-1,3,4-oxadiazole; (6l)}

MP: $1146-148^{\circ} \mathrm{C}$; IR $\left(\mathrm{cm}^{-1}\right): 3060\left(\mathrm{Ar}-\mathrm{H}\right.$ stretch), $2860\left(\mathrm{CH}_{2}\right.$-stretch $), 1668(\mathrm{C}=\mathrm{N}$ stretch of 1,3,4-oxadiazole ring), $2152(\mathrm{C}=\mathrm{C}$ stretching $), 1268(\mathrm{C}-\mathrm{O}-\mathrm{C}$ stretch of 1,3,4-oxadiazole ring), 1090 (C-O stretch); MS: $\mathrm{m} / \mathrm{z}=314 ;{ }^{1} \mathrm{H}$ NMR $\left(\mathrm{CDCl}_{3}\right) \delta$ ppm: $2.30(3 \mathrm{H}, \mathrm{s}), 6.95(1 \mathrm{H}, \mathrm{s})$, 7.08(1H,s), 7.17-7.25 (4H,m), 7.28-7.65 (4H,m); Elemental Analysis: Calcd. For $\mathrm{C}_{17} \mathrm{H}_{12} \mathrm{ClFN}_{2} \mathrm{O} ; \mathrm{C}, 64.87 ; \mathrm{H}, 3.84 ; \mathrm{Cl}, 11.26 ; \mathrm{F}, 6.04 ; \mathrm{N}, 8.90 ; \mathrm{O}, 5.08$ Found: C, 63.88; H, $4.68 ; \mathrm{Cl}, 10.48 ; \mathrm{F}, 6.12 ; \mathrm{N}, 8.98 ; \mathrm{O}, 6.14$

\section{2-(4-butoxystyryl)-5-(4-chlorobenzyl)-1,3,4-oxadiazole; (6m)}

MP: $126-128^{\circ} \mathrm{C}$; IR $\left(\mathrm{cm}^{-1}\right)$ : $3104(\mathrm{Ar}-\mathrm{H}$ stretch $), 2861\left(\mathrm{CH}_{2}\right.$-stretch $), 1665(\mathrm{C}=\mathrm{N}$ stretch of 1,3,4-oxadiazole ring), $2168(\mathrm{C}=\mathrm{C}$ stretching), $1264(\mathrm{C}-\mathrm{O}-\mathrm{C}$ stretch of 1,3,4-oxadiazole ring), $1090(\mathrm{C}-\mathrm{O}$ stretch $) ; \mathrm{MS}: \mathrm{m} / \mathrm{z}=368 ;{ }^{1} \mathrm{H}$ NMR $\left(\mathrm{CDCl}_{3}\right) \delta \mathrm{ppm}: 1.23(3 \mathrm{H}, \mathrm{t}), 1.46$ $(2 \mathrm{H}, \mathrm{m}), 1.68(2 \mathrm{H}, \mathrm{m}), 4.03(2 \mathrm{H}, \mathrm{t}), 6.95(2 \mathrm{H}, \mathrm{m}), 6.98(1 \mathrm{H}, \mathrm{s}), 7.01(1 \mathrm{H}, \mathrm{s}), 7.10-7.24(4 \mathrm{H}, \mathrm{m})$, 7.54-7.79 (4H,m); Elemental Analysis: Calcd. For $\mathrm{C}_{21} \mathrm{H}_{21} \mathrm{ClN}_{2} \mathrm{O}_{2} ; \mathrm{C}, 68.38 ; \mathrm{H}, 5.74 ; \mathrm{Cl}, 9.61$; N, 7.59; O, 8.68 Found: C, 68.46; H, 6.44; Cl, 9.28; N, 7.84; O, 9.18

\section{2-(4-chlorobenzyl)-5-(2-nitrostyryl)-1,3,4-oxadiazole; (6n)}

MP: $170-172{ }^{\circ} \mathrm{C}$; IR $\left(\mathrm{cm}^{-1}\right)$ : 3068 (Ar-H stretch), $2864\left(\mathrm{CH}_{2}\right.$-stretch), $1669(\mathrm{C}=\mathrm{N}$ stretch of 1,3,4-oxadiazole ring), $2158(\mathrm{C}=\mathrm{C}$ stretching $), 1310(\mathrm{C}-\mathrm{O}-\mathrm{C}$ stretch of 1,3,4-oxadiazole ring), 1108 (C-O stretch); MS: $\mathrm{m} / \mathrm{z}=341 ;{ }^{1} \mathrm{H}$ NMR $\left(\mathrm{CDCl}_{3}\right) \delta \mathrm{ppm}: 3.80(2 \mathrm{H}, \mathrm{s}), 7.10(1 \mathrm{H}, \mathrm{s})$, $7.18(1 \mathrm{H}, \mathrm{s}), \quad 7.20-7.35(4 \mathrm{H}, \mathrm{m}), 8.29-8.46(4 \mathrm{H}, \mathrm{m})$; Elemental Analysis: Calcd. For $\mathrm{C}_{17} \mathrm{H}_{12} \mathrm{ClN}_{3} \mathrm{O}_{3} ; \mathrm{C}, 59.75 ; \mathrm{H}, 3.54 ; \mathrm{Cl}, 10.37 ; \mathrm{N}, 12.30 ; \mathrm{O}, 14.04$ Found: $\mathrm{C}, 59.68 ; \mathrm{H}, 4.94 ; \mathrm{Cl}$, $11.26 ; \mathrm{N}, 11.48 ; \mathrm{O}, 15.98$

\section{2-(4-chlorobenzyl)-5-(4-ethoxystyryl)-1,3,4-oxadiazole; (6o)}

MP: $136-138^{\circ} \mathrm{C}$; IR $\left(\mathrm{cm}^{-1}\right)$ : $3068(\mathrm{Ar}-\mathrm{H}$ stretch $), 2868\left(\mathrm{CH}_{2}\right.$-stretch $), 1670(\mathrm{C}=\mathrm{N}$ stretch of 1,3,4-oxadiazole ring), $2165(\mathrm{C}=\mathrm{C}$ stretching), $1267(\mathrm{C}-\mathrm{O}-\mathrm{C}$ stretch of 1,3,4-oxadiazole ring), 1102 (C-O stretch); $\mathrm{MS}: \mathrm{m} / \mathrm{z}=340 ;{ }^{1} \mathrm{H}$ NMR $\left(\mathrm{CDCl}_{3}\right) \delta \mathrm{ppm}: 1.30(3 \mathrm{H}, \mathrm{t}), 1.56(2 \mathrm{H}, \mathrm{q})$, $3.95(2 \mathrm{H}, \mathrm{s}), 6.78(1 \mathrm{H}, \mathrm{s}), 7.04(1 \mathrm{H}, \mathrm{s}), 7.16-7.21 \quad(4 \mathrm{H}, \mathrm{m}), 7.62-7.70 \quad(4 \mathrm{H}, \mathrm{m})$; Elemental Analysis: Calcd. For $\mathrm{C}_{19} \mathrm{H}_{17} \mathrm{ClN}_{2} \mathrm{O}_{2} ; \mathrm{C}, 66.96 ; \mathrm{H}, 5.03 ; \mathrm{Cl}, 10.40 ; \mathrm{N}, 8.22 ; \mathrm{O}, 9.39$ Found: $\mathrm{C}$, 67.26; H, 8.04; Cl, 9.48; N, 8.84; O, 10.26 


\section{RESULT AND DISCUSSION}

Table 1. Optimization of time and yield for the microwave assisted synthesis of compound $6 \mathrm{c}$ and $6 \mathrm{~d}$ using different reagents.

\begin{tabular}{|c|c|c|c|}
\hline Entry as & Reagents & $\begin{array}{c}\text { Time } \\
\text { (min.) }\end{array}$ & Yield \% \\
\hline $6 c$ & $\mathrm{POCl}_{3} / \mathrm{ZnCl}_{2}$ & 5 & 68 \\
\hline 6d & $\mathrm{POCl}_{3} / \mathrm{ZnCl}_{2}$ & 5 & 70 \\
\hline $6 c$ & $\mathrm{POCl}_{3}$ & 8 & 89 \\
\hline 6d & $\mathrm{POCl}_{3}$ & 8 & 85 \\
\hline $6 c$ & $\mathrm{H}_{2} \mathrm{SO}_{4}$ & 13 & 71 \\
\hline 6d & $\mathrm{H}_{2} \mathrm{SO}_{4}$ & 13 & 68 \\
\hline $6 c$ & $\mathrm{H}_{2} \mathrm{SO}_{4} /$ Silica & 10 & 71 \\
\hline 6d & $\mathrm{H}_{2} \mathrm{SO}_{4} /$ Silica & 10 & 76 \\
\hline $6 c$ & $\begin{array}{l}\text { Phosphoric } \\
\text { anhydride }\end{array}$ & 3 & 92 \\
\hline 6d & $\begin{array}{l}\text { Phosphoric } \\
\text { anhydride }\end{array}$ & 3 & 92 \\
\hline $6 c$ & Etidronic acid & 9 & 86 \\
\hline 6d & Etidronic acid & 9 & 86 \\
\hline $6 c$ & PPA & 6 & 74 \\
\hline 6d & PPA & 6 & 74 \\
\hline
\end{tabular}

Table 2. Physical data and Comparison of yield (\%) of the Oxadiazole derivatives obtained using microwave assisted as well as conventional method of synthesis. (Reaction scheme 2).

\begin{tabular}{|c|c|c|c|c|c|c|}
\hline \multirow{2}{*}{ Entry as } & Substitution & \multicolumn{2}{|c|}{$\begin{array}{c}\text { Microwave } \\
\text { Method }\end{array}$} & \multicolumn{2}{|c|}{$\begin{array}{c}\text { Conventional } \\
\text { Method }\end{array}$} & \multirow{2}{*}{$\begin{array}{c}\text { M.P. } \\
\text { ( }\end{array}$} \\
\cline { 2 - 6 } & $\mathbf{R}$ & $\begin{array}{c}\text { Time } \\
\text { (min.) }\end{array}$ & $\begin{array}{c}\text { Yield } \\
\mathbf{\%}\end{array}$ & $\begin{array}{c}\text { Time } \\
\text { (hours) }\end{array}$ & $\begin{array}{c}\text { Yield } \\
\mathbf{\%}\end{array}$ & \\
\hline 6a & $\mathrm{H}$ & 4 & 85 & 8 & 75 & $144-146$ \\
\hline 6b & $2,4-\mathrm{OCH}_{3}$ & 5 & 77 & 8 & 68 & $132-134$ \\
\hline 6c & $4-\mathrm{NO}_{2}$ & 3 & 92 & 8 & 70 & $162-164$ \\
\hline 6d & $4-\mathrm{F}$ & 3 & 92 & 9 & 72 & $140-142$ \\
\hline
\end{tabular}




\begin{tabular}{|c|c|c|c|c|c|c|}
\hline $6 e$ & $4-\mathrm{OCH}_{3}$ & 4 & 76 & 7 & 64 & $136-138$ \\
\hline $6 f$ & $4-\mathrm{CH}_{3}$ & 4 & 81 & 9 & 72 & $130-132$ \\
\hline $6 \mathrm{~g}$ & $4-\mathrm{OC}_{8} \mathrm{H}_{15}$ & 6 & 76 & 9 & 63 & $120-122$ \\
\hline $6 \mathrm{~h}$ & $4-\mathrm{Cl}$ & 4 & 85 & 8 & 71 & $138-140$ \\
\hline $6 \mathbf{i}$ & $2,5-\mathrm{OCH}_{3}$ & 6 & 72 & 9 & 64 & $124-126$ \\
\hline $6 \mathbf{j}$ & $3,4,5-\mathrm{OCH}_{3}$ & 6 & 80 & 8 & 60 & $120-122$ \\
\hline $6 k$ & $\begin{array}{c}-\mathrm{OCH}_{3} \\
4-\mathrm{OH}\end{array}$ & 6 & 79 & 9 & 62 & $142-144$ \\
\hline 61 & $2-F$ & 4 & 85 & 8 & 73 & $146-148$ \\
\hline $6 m$ & $4-\mathrm{OC}_{4} \mathrm{H}_{9}$ & 5 & 76 & 9 & 62 & $126-128$ \\
\hline $6 n$ & $2-\mathrm{NO}_{2}$ & 4 & 87 & 8 & 69 & $170-172$ \\
\hline 60 & $4-\mathrm{OC}_{2} \mathrm{H}_{5}$ & 5 & 83 & 8 & 70 & $136-138$ \\
\hline
\end{tabular}

Initially, we have synthesized $\mathbf{6 c}$ and $\mathbf{6 d}$ by using different reagents under microwave irradiation. We observed the higher yield within lesser time in the reaction which was carried out using poly phosphoric anhydride as reagent, instead of $\mathrm{POCl}_{3}, \mathrm{POCl}_{3} / \mathrm{ZnCl}_{2}, \mathrm{H}_{2} \mathrm{SO}_{4}$, and $\mathrm{H}_{2} \mathrm{SO}_{4}$ /Silica, Etidronic acid (bis phosphoric acid), PPA. The time taken for the completion of reaction was 3 to 13 minutes and the \% yields observed was $68-92 \%$ under microwave assisted method. There for we carried out the same synthesis using phosphoric anhydride as catalyst under conventional method. The time taken for synthesis of all 6a-o compounds by conventional method was about 7-9 hours and the \% yield observed was about $60-75 \%$. All the synthesized compounds were characterized by TLC, elemental analysis, IR ${ }^{1} \mathrm{H}$ NMR and ${ }^{13} \mathrm{C}$ NMR.

\section{CONCLUSION}

In conclusion, the microwave assisted procedure is more convenient for the synthesis of 2,5-di-substituted 1,3,4-oxadiazole derivatives. The virtues of procedure are proficient methodology, excellent yields, safe and an environmentally benign technique with reduces the reaction time, simple work-up method and getting pure product in quantitative yields and gives the opportunity to increase the work flow.

\section{ACKNOWLEDGEMENTS}

The authors are thankful to Department of Chemistry (DST-FIST Funded \& UGC-SAP Sponsored), Saurashtra University, Rajkot and specially indebted to "National Facility for Drug Discovery through New Chemical Entities (NCE's), Development \& Instrumentation Support to Small Manufacturing Pharma Enterprises", a programme under Drug \& Pharma Research Support (DPRS) jointly funded by Department of Science \& Technology, New Delhi, Government of Gujarat (Industries Commissioner ate) \& Saurashtra University, Rajkot. 


\section{Referances}

[1] Potts, K. In Compr. Heterocyclic Chem.; Katritzky, A.R., Rees, Ch., Eds.; Pergamon Press: NY; 6 (1984) 427.

[2] Kulkarni Y. D., Rowhani A., J. Indian Chem. Soc. 66 (1989) 492.

[3] Mohamed Ashraf Ali, Mohammad Shaharyar, Bioorganic \& Medicinal Chemistry Letters 17 (2007) 3314-3316.

[4] Neelam Jain, D. P. Pathak, Pradeep Mishra, Sandeep Jain, Der Pharmacia Lettre, 5 (2013) 415-418.

[5] S. L. Gaonkar, K. M. L. Rai, B. Prabhuswamy, Eur. J. Med. Chem. 41 (2006) 841-846.

[6] Zampieri D., et al., Bioorg. Med. Chem. 17 (2009) 4693-4707.

[7] B. Chandrakantha, Prakash Shetty, Vijesh Nambiyar, Nishitha Isloor, Arun M. Isloor, European Journal of Medicinal Chemistry 45 (2010) 1206-1210.

[8] Samir Bondock, Shymaa Adel, Hassan A. Etman, Farid A. Badria; European Journal of Medicinal Chemistry 48 (2012) 192-199.

[9] Rajesh A. Rane, Pavankumar Bangalore, Sheetal D. Borhade, Preeti K. Khandare, European Journal of Medicinal Chemistry 70 (2013) 49-58.

[10] Jansen M., et al., H. J. Med. Chem. 51 (2008) 4430-4448.

[11] P. C. Unangast, G. P. Shrum, D. T. Conner, C. D. Dyer, D. J. Schrier, J. Med. Chem. 35 (1992) 3691-3698.

[12] V. Ravichandran, S. Shalini, K. Sundram, A. Dhanaraj Sokkalingam, European Journal of Medicinal Chemistry 45 (2010) 2791-2797.

[13] Omar F. A., Mahfouz N. M., Rahman, M. A., Eur. J. Med. Chem.Chim. Ther. 31 (1996) 819.

[14] Dipti L. Namera, Jaynt B. Rathod, Rupali H. Maheta, Umed C. Bhoya, International Letters of Chemistry, Physics and Astronomy 10 (2014) 46-54. 